\title{
Some Properties of Nearly Premixed Laminar Flame Propagation Along Weakly Stratified Layers in Combustible Gas Mixtures
}

\author{
D. G. TALLEY \\ Department of Mechanical Engineering and Applied Mechanics, The University of Michigan, \\ Ann Arbor, MI 48109-212I
}

\begin{abstract}
Nearly premixed laminar flame propagation along weakly stratified layers in combustible gas mixtures is examined theoretically in the limit of weak flame stretch, high activation energy, and unity Lewis number. The stratification is represented by the variation in flame temperature along the reaction zone. Stratification is shown to affect the propagation velocity by a convective loss mechanism and by a diffusive mechanism that could be termed flame thickening. Whether a given amount of stratification should be expected to increase or decrease the propagation velocity compared with that of a nonstratified mixture depends on the angle the flame makes to the flow. The propagation velocity is most sensitive to the stratification for small flame angles.
\end{abstract}

\section{INTRODUCTION}

Laminar flames have been studied for many years. Two limiting cases that have been the most frequently analyzed are premixed flames, in which all reactants are completely mixed prior to combustion, and diffusion flames, in which the reactants are initially completely separated. Premixed flames have associated with them the concept of a propagation velocity; diffusion flames normally do not, although propagation can occur along the interface between air and fuel layers such as sometimes occurs along the roofs of mine shafts [1, 2]. Between these two extremes, an entire spectrum exists in which the reactants are neither completely premixed nor fully separated prior to combustion. Such situations can arise, for example, in the dropwise propagation of flames through sprays, in the propagation of flames over volatile liquid fuel surfaces, at the leading edge of lifted turbulent diffusion flames, and in many practical applications. Compared with the two better-known limiting cases, these intermediate mixtures have so far received relatively little attention.

If the unburned reactants are significantly unmixed over identifiable mean length and time scales, the mixture will be said here to be stratified. This definition serves to eliminate random variations in concentration or temperature due to turbulent fluctuations in otherwise homogeneous flows, local variations resulting from flame instabilities, and variations due to chemical reactions that occur as a normal result of flame passage. Although this definition seems sufficient for present purposes, modification may be necessary in specific applications. Flame propagation is said to be parallel to the stratification if its direction is parallel to the vector gradient of the quantity (for instance, fuel concentration) that is stratified. It is perpendicular if the direction of propagation is perpendicular to the gradient. Parallel propagation occurs, for example, in flame tubes when the reactants are initially separated in the upper and lower parts of the tube but are allowed to partially interdiffuse prior to passage of the flame. This type of propagation occurs in stratified charge internal combustion engines and has been studied the most frequently [3-6]. Although the issues are not fully resolved, there are indications of faster overall burning times and wider flammability limits than in the equivalent homogeneous mixtures. Perpendicular propagation can occur along fuel-air 


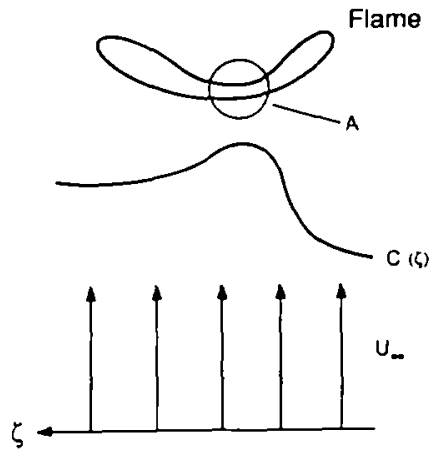

Fig. 1. Flame propagating through stratified mixture.

interfaces, as in the examples given above. An intriguing aspect of the latter kind of propagation is that the velocity of the flame front can be several times faster than that of the stoichiometrically premixed fuel-air mixture $[1,2]$.

One possible mode of perpendicular propagation is illustrated in Fig. 1. A flame propagates steadily into a combustible mixture that is supplied at a uniform velocity $U_{\infty}$. A stratification $C(\zeta)$ in the concentration of some reactant, inert, or in the upstream temperature exists just upstream of the flame transverse to the flow such that the flame becomes gradually weaker and perhaps extinguishes toward the ends. As a consequence of the stratification, the flame becomes curved and its thickness varies with $\zeta$. For simplicity, secondary reactions behind the flame are assumed here not to occur.

Of fundamental interest is the propagation velocity of such a front. This is known to be influenced by several factors. For instance, gas expansion behind the flame can increase the flame velocity in cases where the mixture ahead of the flame is flammable only in narrow regions by altering the flow there [1,7]. This effect does not depend on the flame structure and can be explained aerodynamically by assuming the flame to be an infinitesimally thin surface of discontinuity. In addition, the flame front curvature can cause the streamlines of the flow to diverge, and an increase in propagation velocity can also be predicted in part by aerodynamic considerations alone without reference to flame structure [2]. However, the divergence in streamlines also causes flame stretch, and the flame front curvature can affect the diffusion of heat and mass, depending on the Lewis num- ber. These effects are known to have a fundamental impact on the structure of the flame that may not always positively influence the propagation. Finally, stratification can externally impose variations in temperature and composition along the flame that would not otherwise be present. These would be expected to influence the propagation by generating secondary fluxes of heat and chemical species parallel to it. This latter effect forms the subject of this paper.

Attention is restricted to the isolated impact that secondary heat fluxes have on the flame propagation. No attempt has been made to include the interaction of the stratification with the other factors mentioned above; that is left to later work. Consequently, aerodynamic effects are minimized and Lewis number effects are not considered. Flame curvature, instability, and feedback mechanisms are also neglected, except that stretch is allowed in terms of a weak upstream velocity gradient, which is assumed known. The minimization of these factors amounts to a weak stratification limit whereby the flame is considered to be nearly premixed and to possess most of the features of premixed flame propagation. The details of the theory are presented below.

\section{THEORY}

In their original analysis, Karlovitz et al. [8] first isolated and demonstrated the theoretical importance of flame stretch by considering the idealized flame geometry of Fig. 2, except that here a variation $T_{\mathrm{f}}(\zeta)$ in the reaction zone temperature along the flame has been added as shown, which was not originally allowed. The velocity component normal to the flame decreases as the flame coordinate $\eta$ decreases as a result of the positive velocity gradient $d U_{\infty} / d y$. Continuity then requires that the parallel component of velocity increase, generating stretch. For a thin, locally flat reaction zone at a flame angle $\phi$, parallel streamlines, and constant $T_{\mathrm{f}}(\zeta)$, Karlovitz et al. showed that when the velocity field is expanded to first order using a Taylor series about $\zeta=0$, the governing thermal equation in the preheat zone becomes

$k \frac{\partial^{2} T}{\partial \eta^{2}}+\rho_{\infty} C_{\mathrm{p}} S(1+a \eta) \frac{\partial T}{\partial \eta}=0$, 


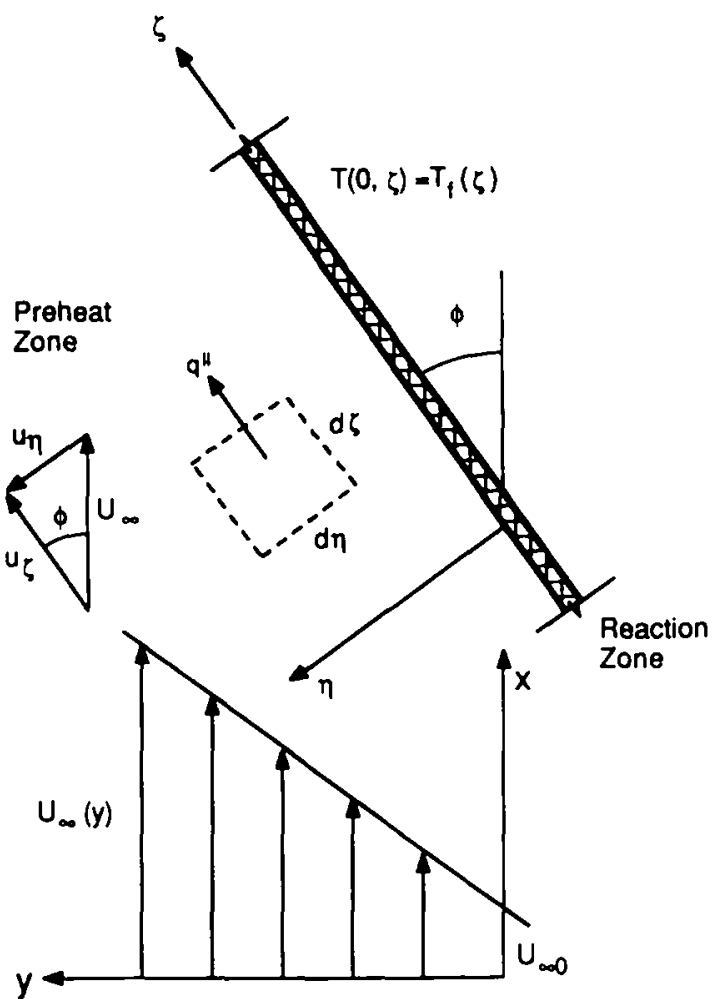

Fig. 2. Combustion wave in a flow with velocity gradient and reaction zone temperature gradient.

where $k$ and $C_{\mathrm{p}}$ are constant, $S$ is the propagation velocity at $\zeta=0$, and

$a \equiv \frac{\cos \phi}{U_{x 0}} \frac{d U_{\infty}}{d y}$,

the flame stretch in this geometry. This analysis corresponds most closely to limitingly small amounts of stretch. Solution of Eq. 1 demonstrated that stretch can reduce the propagation velocity if thermal effects dominate, due to the heat convected out of the preheat zone parallel to the flame by the stretched velocity field.

The goal here will be to introduce a small amount of stratification into the geometry of Fig. 2. However. in order to fix concepts a simpler case will be considered first in which the flame is locally horizontal $(\phi=\pi / 2)$ with zero stretch, as illustrated in Fig. 3. Such a geometry might correspond to the region marked $\mathrm{A}$ in Fig. 1.

The steady flame to which $(\eta, \zeta)$ coordinates are attached is supplied with fresh combustible

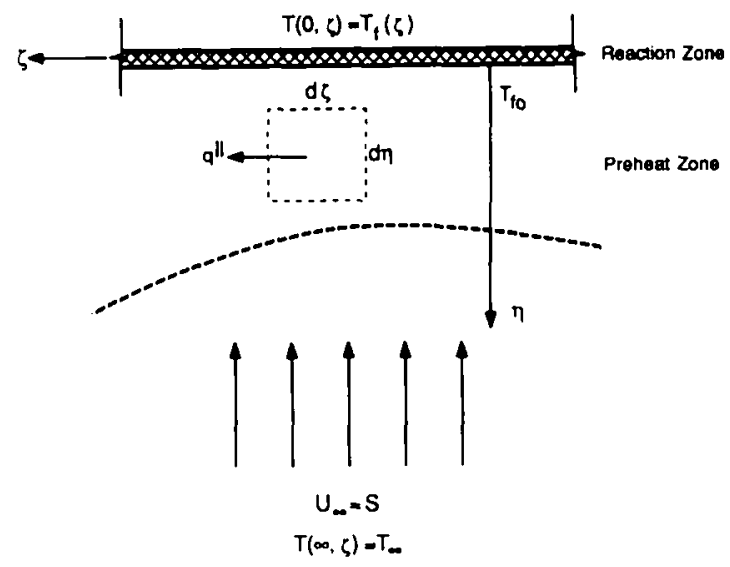

Fig. 3. Simplified geometry.

mixture at uniform upstream temperature $T_{\infty}$ and uniform velocity $U_{\infty}$ equal to the flame propagation velocity $S$. The flame is assumed to possess a two-zone structure consisting of a preheat zone and a reaction zone. Chemistry is assumed to consist of the first-order unimolecular reaction $\mathbf{R} \rightarrow P$ with reaction rate equal to $B Y_{\mathrm{r}} e^{-E / R T} \mathrm{~g} /\left(\mathrm{cm}^{3} \cdot \mathrm{s}\right)$, where $Y_{\mathrm{r}}$ is the mass fraction of reactant and $B$ is assumed constant. The activation energy $E$ is assumed to be large, so the reaction zone can be considered infinitesimally thin over the region of interest. For unity Lewis number, the flame structure can be described using a thermal theory, and under these conditions the effect of stratification can be introduced by allowing the flame temperature to vary along the reaction zone as $T_{f}(\zeta)$.

Since $u_{\zeta}=0$ in this case, the energy equation guverning the preheat zone can be written as

$\rho C_{\mathrm{p}} u_{\eta} \frac{\partial T}{\partial \eta}=k \frac{\partial^{2} T}{\partial \eta^{2}}-\frac{\partial q^{\|}}{\partial \zeta}$

where $q^{\|} \equiv-k(\partial T / \partial \zeta)$ is the component of the heat flux conducted parallel to the flame. For an infinitesimally thin reaction zone, the heat released by the reaction zone into the preheat zone depends only on the local flame temperature and not on the gradient $d T_{\mathrm{r}} / d \zeta$. Consequently, if $S_{\mathrm{u}}$ is the propagation velocity of a one-dimensional nonstratified flame at temperature $T_{\mathrm{f}}$, the heat released by the stratified flame at local temperature $T_{\mathrm{f}}$ is given by $\rho_{\infty} C_{\mathrm{p}} S_{\mathrm{u}}\left(T_{\mathrm{f}}-T_{\infty}\right)$. This consti- 
tutes a boundary condition prescribing the heat that must be conducted into the preheat zone for the stratified case. At $\zeta=0$, the corresponding values are denoted $S_{\mathrm{u} 0}$ and $T_{\mathrm{f} 0}$. Defining the dimensionless variables

$\theta \equiv\left(T-T_{\infty}\right) /\left(T_{\mathrm{f} 0}-T_{\infty}\right), \quad z \equiv \rho_{\infty} C_{\mathrm{p}} S_{\mathrm{u} 0} \eta / k$

$\xi \equiv \rho_{\infty} C_{\mathrm{p}} S_{\mathrm{u} 0} \zeta / k, \quad \lambda=S / S_{\mathrm{u} 0}$,

and

$q \equiv \frac{q^{\|}}{\rho_{\infty} C_{\mathrm{p}} S_{\mathrm{u} 0}\left(T_{\mathrm{fo}}-T_{\infty}\right)}$,

the energy equation, with $\rho u_{\eta}=-\rho_{\infty} S$ by continuity, becomes

$\frac{\partial^{2} \theta}{\partial z^{2}}+\lambda \frac{\partial \theta}{\partial z}=q_{\xi}$

where $q_{\xi}$ denotes the derivative of $q$ with respect to $\xi$. Physically, the term $k / \rho_{\infty} C_{\mathrm{p}} S_{\mathrm{u} 0}$ is a measure of the preheat zone thickness of the nonstratified flame of temperature $T_{\mathrm{f} 0}$, and $\lambda$ is the flame speed eigenvalue. The boundary conditions for Eq. 3 along the line $\xi=0$ become $\theta(0,0)=1$ and $\partial \theta / \partial z(0,0)=-1$. Integrating Eq. 3 along the line $\xi=0$ subject to these boundary conditions gives

$$
\begin{aligned}
\theta(z, 0)= & +\frac{1}{\lambda}\left(e^{-\lambda z}-1\right) \\
& +\int_{0}^{z} e^{-\lambda z}\left(\int_{0}^{z} e^{\lambda z} q_{\xi} d z\right) d z,
\end{aligned}
$$

where $q_{\xi}$ is understood to be evaluated at $\xi=0$. The quantity $\lambda$ is found by letting $\theta \rightarrow 0$ as $z \rightarrow$ $\infty$, giving

$$
\begin{aligned}
\lambda & =1-\int_{0}^{\infty} e^{-\lambda z}\left(\int_{0}^{z} e^{\lambda z} q_{\xi} d z\right) d z \\
& =1-\int_{0}^{\infty} q_{\xi} d z .
\end{aligned}
$$

Equation 4 states that the flame speed is determined by the net inflow or outflow of heat parallel to the flame. For net positive $q_{\xi}$ (outflow), the propagation velocity is reduced. If $q_{\xi}=0, \lambda=1$ and $\theta(z)=e^{-z}, 0 \leq z<\infty$, as usual.

Rigorous evaluation of $q_{\xi}$ depends on the boundary conditions in $\xi$ and integration of $\mathrm{Eq}$. 3 as a partial differential equation. However, in the limit $d T_{\mathrm{f}} / d \zeta \rightarrow 0$ it is possible to adopt a quasi-uniform assumption whereby $q_{\xi}$ can be estimated by assuming the temperature distribution to be locally equal to that of a uniform (nonstratified) distribution defined by the local value of $T_{\mathrm{f}}$. That is, if from Eq. 4 the uniform dimensional temperature distribution for $q_{\xi}=0$ is

$T(\eta)=T_{\infty}+\left(T_{\mathrm{f}}-T_{\infty}\right) \exp \left(-\rho_{\infty} C_{\mathrm{p}} S_{\mathrm{u}} \eta / k\right)$,

then the quasi-uniform temperature distribution, denoted by the subscript $q$, is

$$
\begin{aligned}
T_{\mathrm{q}}(\eta, \zeta)= & T_{\infty}+\left[T_{\mathrm{r}}(\zeta)-T_{\infty}\right] \\
& \times \exp \left[-\rho_{\infty} C_{\mathrm{p}} S_{\mathrm{u}}(\zeta) \eta / k\right]
\end{aligned}
$$

This approximation applies only to the estimation of $q_{\xi}$ for limitingly small values of $d T_{\mathrm{f}} / d \zeta$, not to the actual temperature distribution for the stratified flow. In dimensionless form, this distribution can be written

$\theta_{\mathrm{q}}(z, \xi)=\theta_{\mathrm{f}}(\xi) e^{-\Lambda(\xi) z}$,

where $\theta_{\mathrm{f}}(\xi) \equiv\left[T_{\mathrm{f}}(\xi)-T_{\infty}\right] /\left(T_{\mathrm{fo}}-T_{\infty}\right)$ and $\Lambda(\xi) \equiv S_{\mathrm{u}}(\xi) / S_{\mathrm{u} 0}$, and where $\Lambda(0)=\theta_{\mathrm{f}}(0)=1$. However, since under the present assumptions the nonstratified flame speed $S_{\mathrm{u}}$ depends only on the local flame temperature $T_{\mathrm{f}}$ (see Eq. 14), $\Lambda(\xi)$ can be written equivalently $\Lambda\left[\theta_{\mathrm{f}}(\xi)\right]$. Then approximating $q=-\partial \theta_{\mathrm{q}} / \partial \xi$ and $q_{\xi}=-\partial^{2} \theta_{\mathrm{q}} / \partial \xi^{2}$, one obtains

$$
\begin{aligned}
q & =A e^{-z}+B z e^{-z}, \\
q_{\xi} & =D z e^{-z}+E z^{2} e^{-z},
\end{aligned}
$$

where the constants $A, B, D$, and $E$ are understood to be evaluated at $\xi=0$ and are given by

$A=-\frac{d \theta_{\mathrm{f}}}{d \xi}$,

$B=\frac{d \Lambda}{d \theta_{\mathrm{f}}} \frac{d \theta_{\mathrm{f}}}{d \xi}$, 
$D=\left(\frac{d \theta_{\mathrm{f}}}{d \xi}\right)^{2}\left(2 \frac{d \Lambda}{d \theta_{\mathrm{f}}}+\frac{d^{2} \Lambda}{d \theta_{\mathrm{f}}^{2}}\right)$,

$E=-\left(\frac{d \Lambda}{d \theta_{\mathrm{f}}}\right)^{2}\left(\frac{d \theta_{\mathrm{f}}}{d \xi}\right)^{2}$.

The second derivative $d^{2} \theta_{\mathrm{f}} / d \xi^{2}$ has also been assumed to be negligible in Eq. 8, consistent with the assumption $d \theta_{\mathrm{f}} / d \xi \rightarrow 0$. Substituting Eqs. 7 and 8 into Eq. 5 gives

$$
\begin{aligned}
\lambda & =1-D-2 E \\
& =1+\left(\frac{d \theta_{\mathrm{f}}}{d \xi}\right)^{2}\left[2\left(\frac{d \Lambda}{d \theta_{\mathrm{f}}}\right)^{2}-2 \frac{d \Lambda}{d \theta_{\mathrm{f}}}-\frac{d^{2} \Lambda}{d \theta_{\mathrm{f}}^{2}}\right] .
\end{aligned}
$$

Note that this result admits the possibility of $\lambda$ larger than unity. Resolution of this point depends on $\Lambda\left(\theta_{f}\right)$. This is left to later following further development of the theory.

Returning now to Fig. 2, we consider next the case where the reaction zone temperature is allowed to vary as $T_{\mathrm{f}}(\zeta)$. The same simplifying assumptions are employed as previously, except that a limitingly small velocity gradient $d U_{\infty} / d y$ is also allowed. The governing energy equation becomes

$\rho C_{\mathrm{p}} u_{\eta} \frac{\partial T}{\partial \eta}+\rho C_{\mathrm{p}} u_{\zeta} \frac{\partial T}{\partial \zeta}=k \frac{\partial^{2} T}{\partial \eta^{2}}-\frac{\partial q^{i !}}{\partial \zeta}$.

We seek here, as in Karlovitz et al. [8], the front velocity $S$ at $\zeta=0(y=0)$. Expressing the upstream velocity as $U_{\infty}=U_{\infty 0}+y d U_{\infty} / d y$, then assuming straight streamlines with $\sin \phi=$ $S / U_{\propto 0}$, the normal and parallel velocity components in $(\zeta, \eta)$ coordinates can be written

$\rho u_{\eta}=-\rho_{x} S(1+a \eta+a \zeta \tan \phi)$

and

$\rho u_{\zeta}=\rho_{x} S(1+a \eta+a \zeta \tan \phi) \cot \phi$,

where $a$ is defined following Eq. 1. Taking $\partial T / \partial \zeta=-q^{\|} / k$ and nondimensionalizing as before, one obtains

$$
\begin{aligned}
\frac{\partial^{2} \theta}{\partial z^{2}} & +\lambda(1+\mathrm{Ka} z+\mathrm{Ka} \xi) \frac{\partial \theta}{\partial z} \\
& =q_{\xi}-\lambda(1+\mathrm{Ka} z+\mathrm{Ka} \xi) q \cot \phi
\end{aligned}
$$

where $\mathrm{Ka}$ is the Karlovitz number (三ak) $\left.\rho_{\infty} C_{\mathrm{p}} S_{\mathrm{u} 0}\right)$. Integrating along the line $\xi=0$ with $\theta(0,0)=1$ and $(\partial \theta / \partial z)(0,0)=-1$, one obtains

$$
\begin{aligned}
\theta(z, 0)= & 1-\int_{0}^{z} \exp \left[-\lambda\left(z+\frac{\mathrm{Ka} z^{2}}{2}\right)\right] d z \\
& +\int_{0}^{z} \exp \left[-\lambda\left(z+\frac{\mathrm{Ka} z^{2}}{2}\right)\right] \\
& \times\left\{\int_{0}^{z} \exp \left[+\lambda\left(z+\frac{\mathrm{Ka} z^{2}}{2}\right)\right]\right. \\
& \left.\times\left[q_{\xi}-\lambda(1+\mathrm{Ka} z) q \cot \phi\right] d z\right\} d z,
\end{aligned}
$$

where the eigenvalue $\lambda$ is found implicitly by letting $\theta \rightarrow 0$ as $z \rightarrow \infty$, that is,

$$
\begin{aligned}
1= & \int_{0}^{\infty} \exp \left[-\lambda\left(z+\frac{\mathrm{Ka} z^{2}}{2}\right)\right] d z \\
& -\int_{0}^{\infty} \exp \left[-\lambda\left(z+\frac{\mathrm{Ka} z^{2}}{2}\right)\right] \\
& \times\left\{\int_{0}^{z} \exp \left[+\lambda\left(z+\frac{\mathrm{Ka} z^{2}}{2}\right)\right]\right. \\
& \left.\times\left[q_{\xi}-\lambda(1+\mathrm{Ka} z) q \cot \phi\right] d z\right\} d z
\end{aligned}
$$

Finally, we return to the evaluation of the constants $A, B, D$, and $E$ of Eq. 8 under the quasiuniform assumption, interpreted here to apply in the limit as both the stretch and the stratification approach zero, so that Eqs. 7 and 8 remain valid. The propagation velocity of a nonstratified flame for the first-order unimolecular reaction $\mathbf{R} \rightarrow \mathbf{P}$ has been analyzed asymptotically by Williams [9] for high activation energy. The result was found to first order to be

$$
S_{\mathrm{u}}=\left[\left(\frac{2 k B}{\rho_{\infty} C_{\mathrm{p}}}\right)\left(\frac{R T_{\mathrm{f}}^{2}}{E}\right)^{2} \frac{e^{-E / R T_{\mathrm{f}}}}{\left(T_{\mathrm{f}}-T_{\infty}\right)^{2}}\right]^{1 / 2}
$$

Utilizing this expression to evaluate $\Lambda=S_{\mathrm{u}} / S_{\mathrm{u} 0}$ for the corresponding flame temperatures $T_{\mathrm{f}}$ and 
$T_{\mathrm{fo}}$, the nondimensional result is

$$
\begin{aligned}
\Lambda\left(\theta_{\mathrm{f}}\right)= & \frac{1}{\theta_{\mathrm{f}}}\left[1-\alpha\left(1-\theta_{\mathrm{f}}\right)\right]^{2} \\
& \times \exp \left[-\frac{\beta\left(1-\theta_{\mathrm{f}}\right)}{1-\alpha\left(1-\theta_{\mathrm{f}}\right)}\right],
\end{aligned}
$$

where $\alpha$ and $\beta$ are defined as

$\alpha \equiv\left(T_{\mathrm{fo}}-T_{\infty}\right) / T_{\mathrm{fo}}$

and

$\beta \equiv \frac{E}{2 R_{\mathrm{u}} T_{\mathrm{fo}}}\left(\frac{T_{\mathrm{fo}}-T_{\infty}}{T_{\mathrm{fo}}}\right)$.

Typically, $\alpha \approx 0.5$, while $\beta$, a Zeldovich number, ranges between 3 and 10 . The first and second derivatives $d \Lambda / d \theta_{\mathrm{f}}$ and $d^{2} \Lambda / d \theta_{\mathrm{f}}{ }^{2}$ can be determined from Eq. 15, evaluated at $\xi=0$, where $\Lambda=\theta_{\mathrm{f}}=1$, and used to calculate the constants $A, B, D$, and $E$.

Defining first a stratification number,

$J_{s} \equiv-\beta \frac{d \theta_{\mathfrak{f}}}{d \xi}$,

the results from Eq. 8 are

$$
\begin{aligned}
& A=-J_{\mathrm{s}} / \beta \approx 0, \\
& B=-J_{\mathrm{s}}\left(1+\frac{2 \alpha-1}{\beta}\right) \approx-J_{\mathrm{s}}, \\
& D=J_{\mathrm{s}}{ }^{2}\left[1+\frac{2 \alpha}{\beta}\left(1+\frac{\alpha}{\beta}\right)\right] \approx J_{\mathrm{s}}{ }^{2}, \\
& E=-J_{\mathrm{s}}{ }^{2}\left(1+\frac{2 \alpha-1}{\beta}\right)^{2} \approx-J_{\mathrm{s}}{ }^{2},
\end{aligned}
$$

where the second equalities apply in the limit $\beta \rightarrow \infty$, to be discussed below. These results indicate that the dimensionless propagation velocity $\lambda$, from Eqs. 13, 7, and 19, depends on a stratification number, the Karlovitz number, the Zeldovich number, the flame angle $\phi$, and $\alpha$.

Physically, the limit $\beta \rightarrow \infty$ has meaning for nonstratified flows even for $\beta$ as low as 3 because it appears exponentially inside the reaction rate term [9]. It has somewhat less physical meaning here in terms of the quasi-uniform assumption because it appears nonexponentially in Eq. 19. However, allowing $\beta$ to remain finite would be expected to make only a small quantitative difference in the results to be presented below. Therefore the following results are presented for the distinguished limit $d \theta_{\mathrm{f}} / d \xi \rightarrow 0, \beta \rightarrow \infty$, with $J_{\mathrm{s}}$ remaining finite. Under these conditions,

$$
\begin{aligned}
& q=-J_{\mathrm{s}} z e^{-z} \\
& q_{\xi}=J_{\mathrm{s}}^{2} z e^{-z}(1-z),
\end{aligned}
$$

and $\lambda=\lambda\left(J_{\mathrm{s}}, \mathrm{Ka}, \phi\right)$ only.

\section{RESULTS}

$J_{s}=0$

When the stratification number $J_{\mathrm{s}}$ is zero, the double integral in Eq. 13 becomes zero, the equation becomes independent of $\phi$, and the remaining integral must be evaluated numerically. The results are presented in Fig. 4. Although the above analysis is valid only under conditions of weak stretch, that is, for $\mathrm{Ka} \ll 1$, the plot has been extended here to larger values to better demonstrate the trend. This curve reproduces the original results of Karlovitz et al. [8] and shows that the propagation velocity decreases as the Karlovitz number increases.

$\mathbf{K a}=\mathbf{0}$

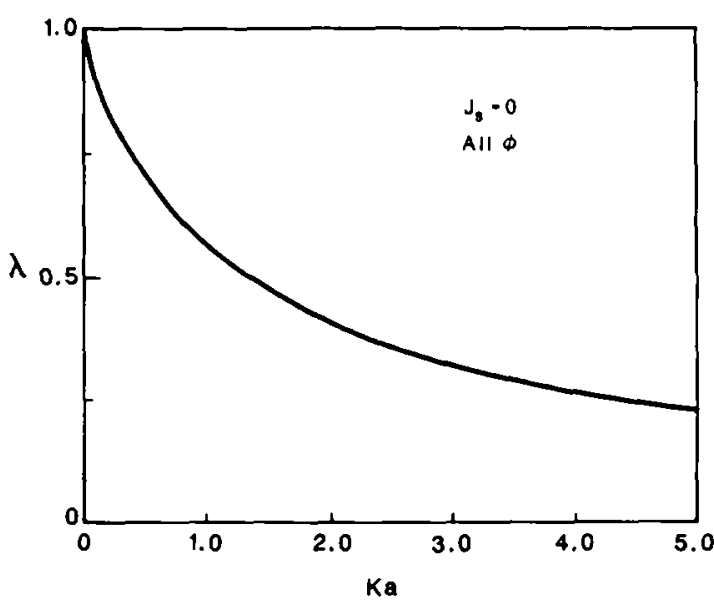

Fig. 4. Results for $J_{s}=0$. 


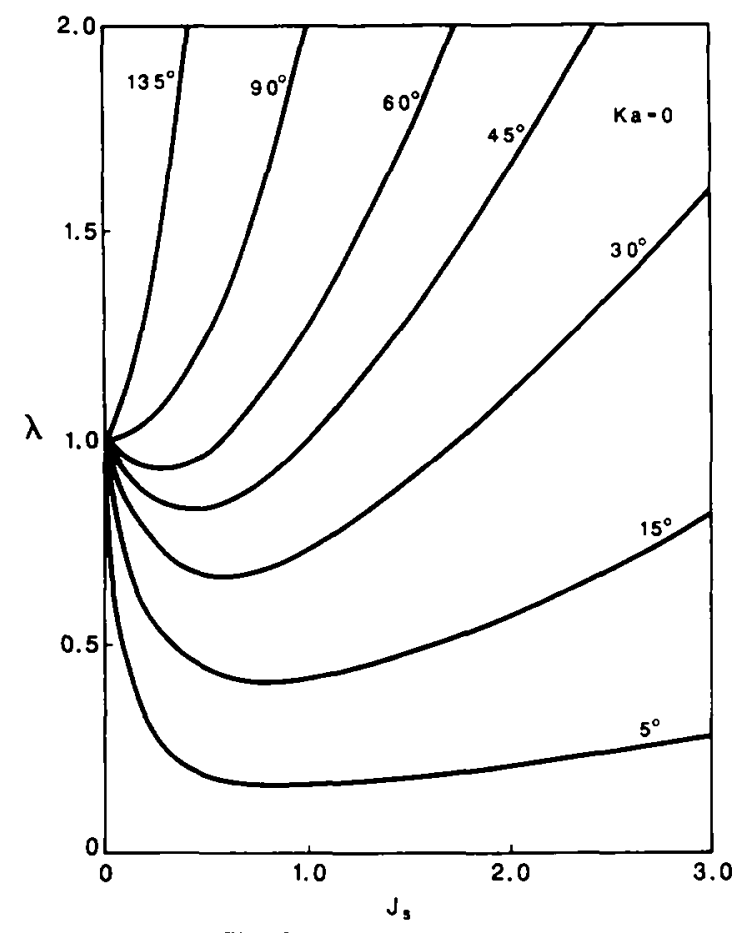

Fig. 5. Results for $\mathrm{Ka}=\mathbf{0}$.

When the Karlovitz number Ka is zero, Eq. 13 can be solved analytically. Substituting Eqs. 20 and 21 into Eq. 13 with $\mathrm{Ka}=0$ gives

$\lambda=\frac{1+J_{\mathrm{s}}{ }^{2}}{1+J_{\mathrm{s}} \cot \phi}$.

Equation 22 is plotted in Fig. 5 for various flame angles $\phi$. Results are shown only for positive values of $J_{s}$; behavior for negative $J_{\mathrm{s}}$ may be deduced by considering the flame angles necessary to reverse the sign of $\cot \phi$. For example, the curve for $\phi=135^{\circ}$ is the reflection about $J_{\mathrm{s}}=0$ of the extension of the curve for $\phi=45^{\circ}$ to negative values of $J_{s}$.

Figure 5 shows that stratification can either increase or decrease the propagation velocity, depending on the flame angle. This can be understood by first referring to the curve for $\phi=90^{\circ}$, corresponding to the geometry of Fig. 2. In this case $\cot \phi=0$, and by comparing it with Eq. 22, Eq. 9 can be shown to reduce to $\lambda=1+J_{\mathrm{s}}{ }^{2}$. The propagation velocity is therefore indeed increased as a result of the stratification, regardless of the sign of $J_{s}$. The reason for this can be found by referring to Eq. 5, where it was remarked that the propagation velocity must be reduced if $q_{\xi}$ is net positive over the preheat zone. However, according to Eq. $21, q_{\xi}$ is negative for $z>1$ and is nonnegative only in the interval $0 \leq z \leq 1$. The quantity $q_{\xi}$ is therefore net negative in the preheat zone, and $\lambda$ must be greater than unity. Physically, this is a diffusive effect that results from the variation in preheat zone thickness along the flame. In the presence of a gradient in reaction zone temperature, the gas flowing into the flame in the local preheat zone along the line $\xi=0$ is surrounded by immediately adjacent flows of lower and higher reaction zone temperatures. It turns out that the flow with the lower reaction zone temperature actually causes more hot fluid than cold fluid to come into contact with the central preheat zone because the preheat zone of the flow with the lower reaction zone temperature is longer. The opposite effect occurs for the hotter reaction zone flow, but because the preheat zone thickness varies exponentially with reaction zone temperature, the effect is not so severe. The result is a net influx of heat into the central preheat zone that does not depend on the direction of the stratification. This phenomenon might be termed flame thickening.

For flame angles different from $90^{\circ}$, this effect competes with a convective effect that might be termed convective loss. It is different from the convective heat loss caused by flame stretch because it occurs here for zero Karlovitz number. It can be explained in terms of the interaction of the velocity component parallel to the flame with the temperature gradients along it. For small flame angles and positive $J_{\mathrm{s}}$, more heat is initially convected out of the central preheat zone at $\xi=0$ than is diffused in by flame thickening, resulting in a lower propagation velocity. As $J_{\mathrm{s}}$ is increased, a critical value is reached where the effect of flame thickening becomes dominant. For flame angles greater than $90^{\circ}$, however, this competition is absent, and the two effects are always additive. Convective loss therefore depends on the direction of stratification (or equivalently, on the sign of $\cot \phi$ ). To summarize, whether stratification causes the propagation velocity to increase or 


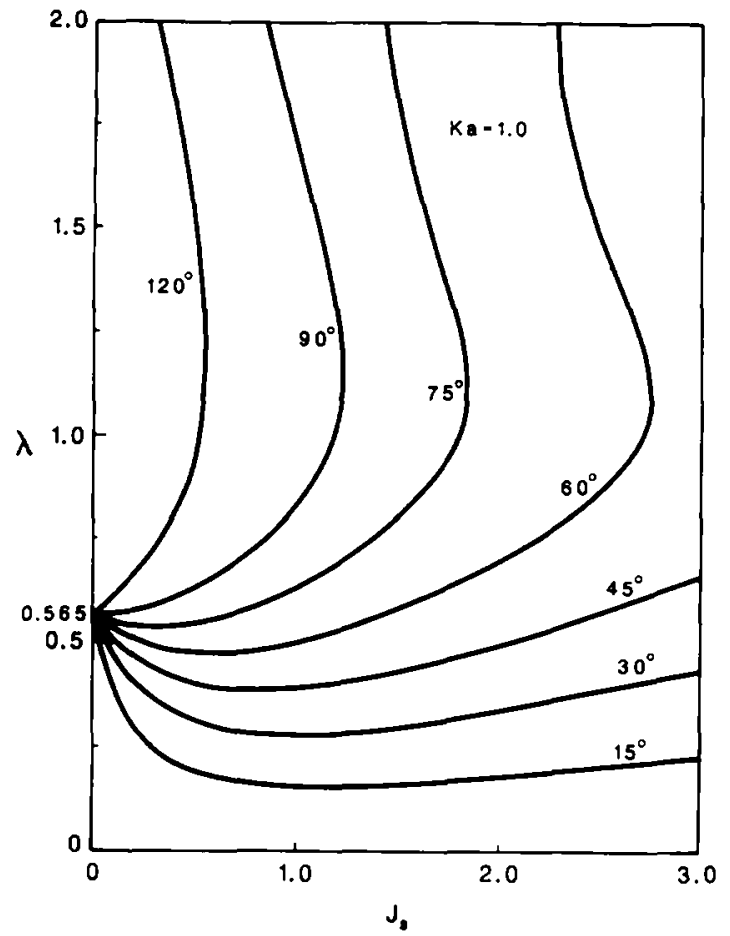

Fig. 6. Results for $\mathrm{Ka}=1.0$.

decrease compared to a nonstratified flow depends on the relative dominance of flame thickening and convective loss for a given flame angle $\phi$.

\section{$J_{\mathbf{s}} \neq \mathbf{0} ; \mathbf{K a} \neq \mathbf{0}$}

In this case, Eq. 13 must be integrated numerically. The results are presented in Figs. 6 and 7 for $\mathrm{Ka}=1$ and $\mathrm{Ka}=2$, respectively. As with Fig. 4, large Karlovitz numbers have been used, even though the analysis is valid only for $\mathrm{Ka} \ll 1$, in order to more clearly demonstrate the trends. The numerical method was checked against the case $\mathrm{Ka}=0$ and was found to adequately reproduce the results of Eq. 22. All curves converge to a single value at $J_{\mathrm{s}}=0$, which corresponds to the curve of Fig. 4 for the appropriate value of Ka. As before, the results for negative values of $J_{\mathrm{s}}$ can be deduced by reflecting the curves for flame angles that reverse the sign of $\cot \phi$.

These figures incorporate the convective effect of flame stretch into the propagation behavior. However, aside from the fact that $\lambda \neq 1$ when

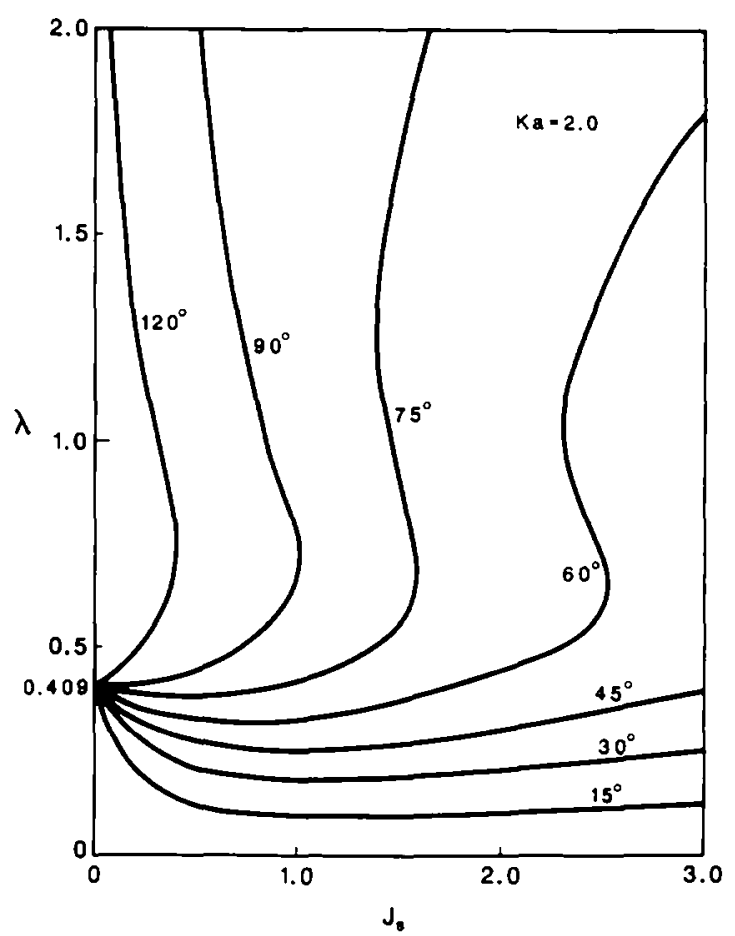

Fig. 7. Results for $\mathrm{Ka}=2.0$.

$J_{s}=0$, the major qualitative difference from Fig. 5 is the existence of multiple roots at sufficiently high values of $J_{\mathrm{s}}$. These may be an artifact of the quasi-uniform approach, since at finite values of $\beta$ this approach applies only at $J_{\mathrm{s}} \rightarrow 0$. For large $J_{s}$, other nonlinear effects not considered in the idealized geometry presented here could significantly alter the shapes of the curves. However, if this effect is indeed real, it is probable that only the lower roots are stable and that stable solutions may not exist for values of $J_{\mathrm{s}}$ beyond certain critical values. The competing influence of flame thickening and convective loss remains evident in these figures.

\section{ZEROTH-ORDER KINETICS}

The above analysis has been repeated for zerothorder kinetics where the reaction rate is equal simply to $B e^{-E / R T}$. This affects the results by changing the functional dependence of $\Lambda\left(\theta_{f}\right)$. For high activation energy, the expression for the onedimensional propagation velocity of the nonstrati- 
fied flame to first order leads to the expression

$$
\begin{aligned}
\Lambda\left(\theta_{\mathrm{f}}\right)= & \frac{1}{\theta_{\mathrm{f}}^{1 / 2}}\left[1-\alpha\left(1-\theta_{\mathrm{f}}\right)\right] \\
& \times \exp \left[-\frac{\beta\left(1-\theta_{\mathrm{f}}\right)}{1-\alpha\left(1-\theta_{\mathrm{f}}\right)}\right],
\end{aligned}
$$

whereupon the constants $A, B, D$, and $E$ of Eq. 8 become

$$
\begin{aligned}
& A=-J_{\mathrm{s}} / \beta, \\
& B=-J_{\mathrm{s}}\left[1+\frac{(2 \alpha-1)}{2 \beta}\right], \\
& D=J_{\mathrm{s}}{ }^{2}\left[1+\frac{1}{\beta}+\frac{1}{\beta^{2}}\left(\alpha-\frac{1}{4}\right)\right], \\
& E=-J_{\mathrm{s}}{ }^{2}\left[1+\left(\frac{2 \alpha-1}{2 \beta}\right)\right]^{2} .
\end{aligned}
$$

These expressions, although slightly different from Eqs. 19, reduce to identically the same limit as $\beta \rightarrow \infty$ with $J_{s}$ finite. Therefore the essential trends identified for first-order kinetics apply also to zeroth-order kinetics.

\section{DISCUSSION}

The analysis presented here has served to isolate the purely thermal effects of stratification in the limit of weak stratification, weak stretch, large activation energy, and unity Lewis number and, practically speaking, to small $J_{\mathrm{s}}$ when $\beta$ is finite. Despite these severe restrictions, it is nevertheless possible to extract several interesting features. First is the central role played by the stratification number $J_{\mathrm{s}}$ as defined by Eq. 18 . By replacing the variables in Eq. 18 with dimensional quantities, it is possible to interpret the physical meaning of this number. The result can be expressed as

$$
J_{\mathrm{s}}=\beta\left(q_{\mathrm{f}}{ }^{\prime \prime} / q_{\mathrm{f}}^{\perp}\right),
$$

where $q_{\mathrm{f}}{ }^{\prime \prime}=-k d T_{\mathrm{f}} / d \zeta$ is the heat flux parallel to the flame at the reaction zone and $q_{\mathrm{f}}{ }^{\perp} \equiv \rho_{\infty} C_{\mathrm{p}} S_{\mathrm{u} 0}\left(T_{\mathrm{fo}}-T_{\infty}\right)$ is the perpendicular heat flux at the reaction zone. The stratification number is seen to be the ratio of these two fluxes multiplied by the Zeldovich number $\beta$, which can be interpreted as a measure of the temperature sensitivity of the reaction rate.

A second feature is the identification of two thermal mechanisms through which stratification influences the flame propagation; convective loss, different from the loss caused by flame stretch; and a diffusive loss associated with flame thickening. Convective loss takes on special importance at small flame angles, where examination of Figs. 4-6 shows the behavior to be extremely sensitive to stratification even for small values of $J_{\mathrm{s}}$. One instance in which small flame angles would be expected to arise is near extinction in highly stretched velocity fields, such as near the blowout of premixed jet flames. Since significant concentration gradients as well as velocity gradients would be expected to occur near the exit nozzle of such flames, the results suggest that stratification could play as significant a role as stretch in determining the eventual blowout behavior of these flames. Finally, it should be recalled that the thermal effects presented here are only two of a much wider range of coupled effects that would be expected to occur in reality, as discussed in the Introduction. More complete investigation of these effects should provide fruitful topics for future research.

\section{CONCLUSIONS}

An analysis has been presented in which the purely thermal effects of stratification in a mixture upstream of a nearly premixed laminar flame have been investigated in the limit of weak stratification, weak stretch, high activation energy, and unity Lewis number for zeroth- and first-order unimolecular reactions. The results are found to depend on the Karlovitz number Ka, the Zeldovich number $\beta$, the flame angle $\phi$, and a stratification number $J_{\mathrm{s}}$ defined by Eq. 18 . The thermal effects of stratification have been identified in terms of a convective loss mechanism, different from stretch, and a diffusive mechanism associated with flame thickening. It is found that the propagation velocity can be either increased or decreased depending on the flame angle and that the results become increasingly sensitive to the stratification as the 
flame angle decreases. This suggests that stratification could play as important a role as flame stretch in determining the near-extinction behavior of some flames.

Part of this work was completed while on a Summer Faculty Fellowship at the WrightPatterson Aero Propulsion Laboratory under Air Force contract F49620-87-R-0004.

\section{REFERENCES}

1. Feng, C. C., Lam, S. H., and Glassman, 1., Flame Propagation Through Layered Fuel-Air Mixtures, Combust. Sci. Technol. 10:59-71 (1975).

2. Liebman, I., Corry, J., and Perlee, H. E., Flame Propagation in Layered Methane-Air Systems, Combust. Sci. Technol. 1:257-267 (1970).

3. Liebman, I., Corry, J., and Perlee, H. E., Dynamics of Flame Propagation Through Layered Methane-Air Mixtures, Combust. Sci. Technol. 2:365-375 (1971),.

4. Girard, P., and Leyer, L. C., Constant Volume Combustion of Uniform and Stratified Lean Mixtures of Propane and Air, in Combustion in Reactive Systems (Progress in Astronautics and Aeronautics, Vol. 76), J. R. Bowen et al., Eds., American Institute of Aeronautics and Astronautics, New York, 1979, pp. 565-577.

5. Karim, G. A., and Tzang, P., Flame Propagation Through Atmospheres Involving Concentration Gradients Formed by Mass Transfer Phenomena, J. Fluids Eng. 97:615-617 (1975).

6. Bisset, E. S., and Reuss, D. L., Analysis of a Slowly Varying Nonadiabatic Flame Propagating Through Gradients of Fuel or Temperature, Twenty First Symposium (International) on Combustion, The Combustion Institute, 1985, pp. 531-538.

7. Kulkarny, V. A., Schwartz, J., and Fink, S., A Model of Two-Dimensional Flow Effects in Laser Supported Combustion Waves and Laminar Flames, ALAA-83-1718, 1983.

8. Karlovitz, B., Denniston, D. W., Knapschefer, D. H., and Wells, F. E., Studies on Turbulent Flames, Fourth Symposium (International) on Combustion, The Combustion, Institute, 1953, pp. 613-620.

9. Williams, F. A., Combustion Theory, Benjamin/ Cummings, 1985, p. 159.

Received 5 July 1988; revised 18 November 1988 